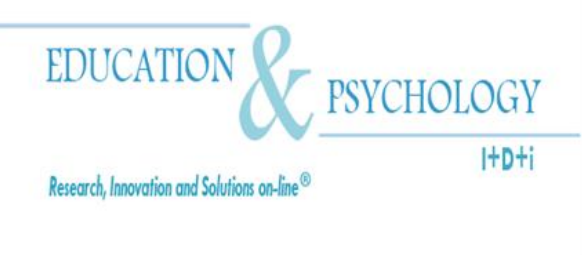

\title{
Validación Psicométrica del Motivated Strategies for Learning Questionnaire en Universitarios Mexicanos
}

\section{María del Carmen Ramírez Dorantes ${ }^{1}$, José Enrique Canto y Rodríguez ${ }^{2}$, José Antonio Bueno Álvarez ${ }^{3}$, Alejandro Echazarreta Moreno ${ }^{1}$}

\begin{tabular}{c}
\hline${ }^{1}$ Dirección General de Desarrollo Académico, \\
Universidad Autónoma de Yucatán \\
${ }^{2}$ Facultad de Psicología, \\
Universidad Autónoma de Yucatán \\
${ }^{3}$ Departamento de Psicología Evolutiva y de la Educación, \\
Universidad Complutense de Madrid
\end{tabular}

México

España

Correspondencia: Ma. Del Carmen Ramírez Dorantes. Dirección General de Desarrollo Académico. Universidad Autónoma de Yucatán. Centro Cultural Unioversitario.Calle 60 No. 491-A X 57 Centro CP 97000. Mérida, Yuc. México. E-mail: marycarmen.ramirez@uady.mx

C Education \& Psychology I+D+i and Editorial EOS (Spain) 


\section{Resumen}

Introducción. El Motivated Strategies for Learning Questionnaire (MSLQ) es un instrumento de autorreporte que mide la motivación y las estrategias de aprendizaje (cognitivas, metacognitivas y de contexto) de los estudiantes. El presente estudio tuvo por objetivo traducir, adaptar y validar el MSLQ al contexto educativo mexicano.

Método. La versión original del MSLQ fue traducida y adaptada al español de México con el nombre de Cuestionario de Motivación y Estrategias de Aprendizaje (CMEA) tomando en cuenta las 22 pautas o directrices que establece la International Test Commission (ITC) y posteriormente administrada a estudiantes $(N=1,140)$ de una universidad del sureste de México.

Resultados. Los resultados permiten concluir que los ítems fueron agrupados en cada uno de los factores mediante la factorización de ejes principales. Los índices de consistencia interna obtenidos con el CMEA fueron aceptables.

Conclusión. Los resultados, muestran una gran potencia del instrumento de proporcionarnos información útil para contar con un instrumento de medida que nos permita encontrar respuesta a la pregunta inicial de los trabajos de autorregulación del aprendizaje: ¿Cómo los estudiantes llegan a ser expertos o desarrollar la destreza de regular sus propios procesos de aprendizaje?

Palabras Clave: MSLQ, propiedades psicométricas, aprendizaje autorregulado, estudiantes universitarios. 


\title{
Psychometric Validation of the Motivated Strategies for Learning Questionnaire, with Mexican University Students
}

\begin{abstract}
Introduction. The Motivated Strategies for Learning Questionnaire (MSLQ) is a self-report instrument designed to assess students' motivation and learning strategies (cognitive, metacognitive, and resource management). In the present study, we focused on translate, adapt and validate the MSLQ to Mexican educational context.

Method. The original version of the MSLQ was translated and adapted to mexican educational context under the name of Cuestionario de Motivación y Estrategias de Aprendizaje (CMEA) taking into account 22 guidelines established by the International Test Commission (ITC) and subsequently administered to mexican students $(N=1,140)$ of a southeastern university of México.
\end{abstract}

Results. Results allow concluding that the items were grouped in each of the factors using factorization of major axes. Internal consistency rates obtained were acceptable.

Discussion and conclusion. The results show great power providing useful information about how this instrument can answer our initial question of self-regulated learning: How do students can become experts in their own learning processess?

Keywords: MSLQ, psychometric properties, self-regulated learning, university students.

Received: $10 / 20 / 12$

Initial acceptance: 02/10/13 Final acceptance: 03/08/13 


\section{Introducción}

Cada vez parece más evidente que la mejora del aprendizaje y del rendimiento académico de los estudiantes, necesariamente tiene que tener en cuenta los componentes cognitivos y motivacionales del aprendizaje, lo que en otras palabras sería afirmar, como lo hicieron Pintrich y De Grott (1990a), que el conocimiento y la regulación de las estrategias cognitivas y metacognitivas estan asociados a que los alumnos estén motivados e interesados por las tareas y actividades académicas. Además, algunos otros investigadores (Paris, Lipson y Wixson, 1983; Pintrich, 1989; Pintrich y De Groot, 1990b; Zimmerman, 2008) consideran prioritario integrar ambos aspectos del aprendizaje si queremos llegar a la elaboración de modelos adecuados sobre el proceso de aprendizaje escolar, ya que los alumnos necesitan tener tanto voluntad (will) como habilidad (skill) de cara a obtener éxitos académicos, y óptimos resultados de aprendizaje.

La interrelación entre la motivación y la cognición fue el tema central en los trabajos del Dr. Paul Pintrich de la Universidad de Michigan, cuya principal contribución a la psicología educativa ha sido su propuesta de un modelo de aprendizaje contextualizado y socialcognitivo como el paradigma dominante. Realizó múltiples y amplias publicaciones acerca de la interrelación entre la "fría" cognición y la "caliente" motivación haciendo un énfasis muy especial en la importancia de la dinámica entre la motivación y la cognición en el desempeño y el aprendizaje durante toda la vida de los estudiantes.

Así mismo, a la prematura muerte del Dr. Paul Pintrich ocurrida en 2004 han aparecido infinidad de artículos en los que se pone de manifiesto la enorme influencia que ejerció y seguirá ejerciendo su obra en la investigación y la práctica educativa (Clearly, Zimmmerman, y Keating, 2006; Dunn, Lo, Mulvenon, y Sutcliffe, 2012; García y McKeachie, 2005; Greene y Azevedo, 2007; Limón, 2004; Núñez, Solano, González-Pienda y Rosario, 2006; Ommundsen, 2006; Schmitz y Wiese, 2006; Schunk, 2005; Schunk y Zimmerman, 2007; Stoeger y Ziegler, 2007; Taylor, 2012; Winne, et al., 2006; Van Den Hurk, 2006; Zimmerman; 2008). Uno de sus grandes legados a la práctica de la psicología educativa y de la investigación empírica acerca del aprendizaje y la motivación en estudiantes universitarios fue el Motivated Strategies for Learning Questionnaire (MSLQ) (Pintrich, Smith, García y McKeachie, 1991; 1993). Un instrumento de medida de autorreporte de 81 reactivos que mide el uso de estrategias de aprendizaje y el nivel de motivación de los estudiantes. Iincorpora aspectos de la autorregulación del aprendizaje en una subescala de autorregulación metacognitiva, la cual enfati- 
za la interrelación entre la motivación y la cognición (Schunk y Zimmerman, 1994; Zimmerman y Schunk, 1989).

El MSLQ ha sido traducido a varios idiomas y usado por cientos de investigadores y profesores alrededor del mundo para enfatizar la naturaleza de la motivación y el uso de estrategias de aprendizaje en distintos tipos de contenido y de poblaciones; para ayudar a refinar el entendimiento teórico de los constructos motivacionales, para explicar cómo son distintos unos de otros, cuáles son las diferencias individuales que existen en la autorregulación del aprendizaje y para evaluar los efectos motivacionales y cognitivos de diferentes aspectos de la instrucción (García y Mckeachie, 2005).

Anterior a la aparición del MSLQ, la mayor parte de las investigaciones acerca del aprendizaje en estudiantes universitarios así como el desarrollo de instrumentos de medición, se concentraba en las diferencias individuales o en los estilos de aprendizaje (introversiónextroversión; dependencia-independencia de campo; perfiles de Myers-Briggs) cuyas relaciones con la conducta de estudio o con el procesamiento cognitivo de los estudiantes no estaba totalmente claro (Lockhart y Schmeck, 1984; Torrance, Reynolds, Riegel y Ball, 1977). Además los inventarios de habilidades de estudio usados en ese momento para medir el aprendizaje del estudiante (Brown y Holtzman, 1967; Christensen, 1968; Goldman y Warren, 1973) fueron criticados de ser ateóricos (Weinstein y Underwood, 1985). Ante este panorama predominante en ese momento, existía una imperiosa necesidad de contar con un instrumento de medición para medir la motivación y las estrategias de aprendizaje que usan los estudiantes.

Ante la cada vez más extendida idea en el contexto educativo de que es el estudiante quien debe establecer sus metas, supervisar y evaluar su desempeño académico, es decir autorregular su aprendizaje y ante la ausencia de instrumentos validos y confiables que sirvan a los propósitos anteriores, se planteó como propósito de esta investigación traducir, desarrollar y reunir información psicométrica inicial acerca del Cuestionario de Motivación y Estrategias de Aprendizaje (CMEA) que es una traducción, adaptación y validación del Motivated Scale Learning Questionnaire (MSLQ) (Pintrich, Smith, García y McKeachie, 1991; 1993).

El MSLQ fue desarrollado usando un punto de vista cognitivo-social de la motivación y las estrategias de aprendizaje desde el cual el estudiante es asumido como un sujeto que 
procesa activamente la información y cuyas creencias y cogniciones son mediadores importantes de la información instruccional y las características de la tarea (García y Pintrich, 1991)

Por otra parte, el punto de vista cognitivo-social en el que el MSLQ está fundamentado asume que la motivación y las estrategias de aprendizaje no son rasgos de los estudiantes pero sí, que la motivación es dinámica y limitada por el contexto y que las estrategias de aprendizaje pueden ser aprendidas y se encuentran bajo el control del estudiante. Es decir, que la motivación de los estudiantes varía en los diferentes cursos (por ejemplo, más interés y valor en un curso elegido libremente y no en un curso obligatorio; más eficacia en un curso fácil de psicología en comparación con curso difícil de matemáticas o física) y que sus estrategias de aprendizaje pueden variar dependiendo de la naturaleza de la tarea académica (examen de elección múltiple v.s. un examen de ensayo) (García y McKeachie, 2005)

Una característica del MSLQ es que fue diseñado enfocándose a un nivel del curso ya que se consideró que el curso era el más apropiado nivel de análisis, que se encuentra situado entre un nivel muy general y global de todas las situaciones y el nivel poco práctico y restringido de cada una de las situaciones específicas de cada curso. Esta operacionalización de la motivación y la cognición también distinguió al MSLQ de otro de los instrumentos de autorreporte ampliamente usados, el Learning and Study Strategies Inventory (LASSI, Weinstein, Schulte y Palmer, 1987) el cual, media las estrategias de aprendizaje y actitudes hacia el estudio en un nivel muy general.

El MSLQ tiene dos secciones: una de motivación y otra de estrategias de aprendizaje. La Escala de Motivación costa de 31 ítems distribuidos en 6 subescalas que miden las metas, las creencias de valor, y de control de pensamientos, las creencias acerca de las habilidades para tener éxito y la ansiedad ante los exámenes. La Escala de Estrategias de Aprendizaje incluye 31 ítems relativos al uso que hacen los estudiantes de diferentes estrategias cognitivas y metacognitivas. Además incluye 19 ítems acerca del manejo de diferentes recursos para el aprendizaje por parte del estudiante, siendo en total 50 ítems distribuidos en 9 subescalas.

La investigación acerca de la autorregulación del aprendizaje académico y el desempeño, surgió hace más de dos décadas para responder la pregunta de cómo los estudiantes llegaban a desarrollar la maestría en sus propios procesos de aprendizaje de tal forma que se ha ido desarrollado gradualmente como resultado del desarrollo de paradigmas teóricos y metodologías diversas (Boekaerts, Pintrich y Zeidner, 2000; Zimmerman y Schunk, 1989, 2001). 
La mayoría de los teóricos de la autorregulación académica conceptualizan el aprendizaje como un proceso multidimensional que involucra componentes personales (cognitivos, motivacionales y emocionales), conductuales y contextuales (Zimmerman, 1986, 1989, 1994). Es decir, para que una habilidad académica llegue a ser ejecutada con maestría, los estudiantes deberán conductualmente, aplicar estrategias cognitivas a una tarea dentro de un contexto determinado. Para lograr esto se requiere de repetidos intentos de aprendizaje, ya que la maestría involucra la coordinación de los componentes personales, conductuales y contextuales o ambientales, cada uno de los cuales es dinámico por sí solo, pero lo son también cuando interactúan conjuntamente. Como resultado de estas condiciones intrapersonales e interpersonales y del contexto, tan diversas y cambiantes, los estudiantes autorregulados deben constantemente revisar su efectividad en el logro de sus metas académicas.

\section{Método}

\section{Participantes}

Los participantes del estudio fueron 1140 estudiantes universitarios matriculados en diferentes facultades y cursando doce diferentes titulaciones en la Universidad Autónoma de Yucatán (UADY). Los estudiantes de la muestra se seleccionaron mediante un procedimiento de muestreo estratificado proporcional con la finalidad de incluir sub-muestras suficientes con grupos de estudiantes de ambos sexos, diferentes titulaciones representativas de las áreas del conocimiento en las que está organizada la UADY y de los cinco cursos en los que están organizados la mayor parte de los programas educativos de las titulaciones. Se solicitó su participación de forma voluntaria para responder al cuestionario asegurándoles la confidencialidad de sus respuestas.

Del total de sujetos 609 (53.4\%) eran mujeres y 531 (46.6\%) eran hombres. El 98\% de la muestra de estudiantes reporto encontrarse en el rango de edad de entre 17 y 55 años con una media de 20.89 años, desviación típica de 2.915 y varianza de 8.498 . Por curso se distribuyeron de la siguiente manera: 272 (23.9\%) de primer curso; 271 (23.8\%) de segundo curso; $282(24.7 \%)$ de tercer curso; 244 (21.4\%) de cuarto curso y 71 (6.2\%) de quinto curso. Por áreas de conocimiento se distribuyeron de la siguiente manera: Ciencias Exactas e Ingeniería, 
255 (22\%), Sociales y Humanidades, 297 (26\%), Ciencias de la Salud, 320 (28\%), Económico- Administrativas y Diseño del Hábitat, 268 (24\%).

Se seleccionó una muestra de estudiantes universitarios debido a que se considera que es en este nivel educativo en el que se produce un mayor control del proceso de aprendizaje por parte de los estudiantes. Es decir, las demandas de este nivel educativo plantean al estudiante universitario mayor control de su motivación, de sus estrategias cognitivas, metacognitivas y de contexto para la obtención de un nivel de logro educativo aceptable.

\section{Instrumento}

Para la conformación de la versión final del instrumento se conservó la estructura teórica de la versión original en inglés, compuesta por 81 ítems distribuidos en 15 subescalas. Por último, se dio forma al cuestionario con los reactivos traducidos y se elaboró un cuadernillo integrado por los 81 reactivos del cuestionario final. Se elaboró también una hoja de respuestas para ser leída por lector óptico para agilizar la fase de captura de información, en la que se incluyó una sección de datos personales, que solicitaba a los estudiantes su edad, sexo y nombre de la titulación. La escala que se utilizó para obtener las respuestas de los estudiantes se realizó en un continuo de siete puntos, igual que en la escala original, en la que 1 significaba "Nada cierto en mí" y 7, "Totalmente cierto en mí". Ver Tabla 1 y 2. La Tabla 1 presenta la distribución de los reactivos de la Escala de Motivación que conforman el instrumento final. La Tabla 2 presenta la distribución de los reactivos de la Escala de Estrategias de Aprendizaje que conforman el instrumento final

Tabla 1. Distribución de los reactivos de acuerdo con su pertenencia a la Escala de Motivación

\begin{tabular}{lcc}
\hline \multicolumn{1}{c}{ Escala de Motivación } & $\mathrm{N}^{\mathbf{o}}$ de reactivo & Total \\
\hline Orientación a metas intrínsecas (OMI) & $1,16,22,24$ & 4 \\
Orientación a metas extrínsecas (OME) & $7,11,13,30$ & 4 \\
Valor de la tarea (VT) & $4,10,17,23,26,27$ & 6 \\
Creencias de control (CC) & $2,9,18,25$ & 4 \\
Autoeficacia para el aprendizaje (AEPA) & $5,6,12,15,20,21,29,31$ & 8 \\
Ansiedad ante los exámenes (AE) & $3,8,14,19,28$ & 5 \\
Total & & 31 \\
\hline
\end{tabular}


Tabla 2. Distribución de los reactivos de acuerdo con su pertenencia a la Escala de Estrategias de Aprendizaje

\begin{tabular}{lcc}
\hline \multicolumn{1}{c}{ Escala de Estrategias de Aprendizaje } & $\mathrm{N}^{\circ}$ de reactivo & Total \\
\hline Repetición (RE) & $39,46,59,72$ & 4 \\
Elaboración (ELA) & $53,62,64,67,69,81$ & 6 \\
Organización (ORG) & $32,42,49,63$ & 5 \\
Pensamiento crítico (PC) & $38,47,51,66,71$ & \\
Autorregulación metacognitiva (ARM) & $33,36,41,44,54,55,56,57,61,76,78$, & 8 \\
& $79,52,65,70,73,77,80$ \\
Administración del tiempo y del am- & $37,48,60,74$ & 4 \\
biente (ATA) & $34,45,50$ & 3 \\
Regulación del esfuerzo (RE) & $40,58,68,75$ & 4 \\
Aprendizaje con compañeros (AC) & & 50 \\
Búsqueda de ayuda (BA) & & \\
Total & & \\
\hline
\end{tabular}

\section{Procedimiento}

La versión original del MSLQ (Motivated Strategies for Learning Questionnaire) fue traducida con autorización del Dr. Pintrich, adaptada y validada al español de México con el nombre de Cuestionario de Motivación y Estrategias de Aprendizaje (CMEA). El procedimiento seguido para la traducción y adaptación del MSLQ se realizó de manera cuidadosa y tomando en cuenta las 22 pautas o directrices que establece la International Test Commission (ITC) para la traducción y adaptación de instrumentos de medida de un idioma a otro y de una cultura a otra (Muñiz y Hambleton, 1996).

Para la traducción y adaptación del instrumento se siguieron los siguientes pasos: inicialmente se procedió a la selección de un equipo de cuatro expertos bilingües mexicanos y se les proporcionó el MSLQ a fin de que realizaran la traducción al español de los 81 ítems que conforman las 15 subescalas del instrumento. Tres de los cuatro expertos eran profesores universitarios y expertos en psicología escolar y profesores y/o investigadores universitarios, con más de 15 años de experiencia en la docencia y en consejo escolar y psicológico con estudiantes de nivel medio superior y superior. Uno de ellos era profesor de enseñanza del inglés y con amplia experiencia en docencia universitaria. En la selección del equipo de tra- 
ductores se cuido que todos ellos no solo conocieran el idioma sino que estuvieran familiarizados con ambas culturas como lo establece la primera directriz que sugiere el ITC. Para asegurar que los traductores tuvieran un cierto conocimiento de la metodología de construcción de test se seleccionó a profesores universitarios que por su experiencia docente habían tenido formación en la elaboración y confección de test y cuestionarios de evaluación. Una vez que el equipo hubo concluido la traducción se procedió a determinar la calidad de la traducción mediante las coincidencias entre ellos. Como resultado de este procedimiento se obtuvieron los 81 ítems traducidos al español.

Una técnica habitual en la traducción de test es lo que se denomina traducción inversa. Para llevar a cabo esta fase de la traducción se seleccionó de nuevo a un equipo de tres expertos traductores angloparlantes y con dominio del español a fin de que volvieran a traducir al idioma original los 81 reactivos. La bondad de la traducción se juzgó en función del grado de coincidencia con la versión original.

De esta fase del proceso de traducción y re-traducción, se concluyó que existían algunos ítems que por su contenido y falta de pertinencia al contexto y nivel educativo en el que se administraría el cuestionario para su validación, no correspondían a la realidad de los estudiantes de la población estudiada, y no representarían conductas significativas para ellos. Como consecuencia de ello se decidió adaptar el contenido de algunos de los reactivos, basándose en la experiencia de la responsable del proyecto y en el conocimiento del contexto educativo de la población estudiada, a fin de hacerlos significativos para ésta. Por ejemplo el reactivo 69 de la subescala de elaboración "Intento entender el material de ésta clase haciendo asociaciones entre las lecturas y los conceptos de las conferencias" se cambió por "Trato de entender el contenido de esta clase, relacionando mis lecturas y los conceptos de las conferencias".

Los cambios que se realizaron en la traducción original de los reactivos, con el propósito de adaptarlos para que fueran usados en la población de estudiantes mexicanos, incluyeron la sustitución de palabras, (por ejemplo, el reactivo 27 de la subescala de valor de la tarea "Entender el tema principal de este curso es muy importante para mi" se sustituyó "Entender el contenido de este asignatura es muy importante para mí”), y traducciones alternativas, (por ejemplo, el reactivo 24 de la subescala de orientación a metas intrínsecas "Cuando tenga la oportunidad en esta clase, escogeré cursos de los cuales pueda aprender, incluso si no me 
garantizan obtener una buena calificación" fue adaptado como "Cuando tenga la oportunidad en este curso elegiré tareas o actividades que me permitan aprender cosas nuevas aunque no me garanticen buenas calificaciones", cuyo contenido está más acorde con las circunstancias escolares de la población estudiada, y en esencia reproduce el contenido del ítem original.

Una vez llevados a cabo los pasos anteriores se decidió explorar la validez de contenido de los ítems ya traducidos para lo cual, se les proporcionó a tres profesores universitarios el cuestionario y se les solicito su valoración respecto a si consideraban que cada uno de los ítems pertenecía a la subescala original. Se les proporciono la definición conceptual de cada una de las escalas para guiar su decisión. En términos generales los tres profesores coincidieron en que los reactivos median el constructo de la subescala a la que pertenecían.

Como resultado de todo el procedimiento anterior se obtuvo el cuestionario traducido y listo para ser probado en una fase piloto con una muestra de estudiantes universitarios. El propósito del estudio piloto fue documentar (a) la claridad de las instrucciones, de los reactivos y la hoja de respuestas, y (b) el nivel de comprensión de los enunciados de los ítems.

Para la administración del instrumento en la fase piloto del estudio, se seleccionó una muestra aleatoria simple de 80 estudiantes de la carrera de psicología de la UADY, integrada por 60 mujeres y 20 hombres, cuyas edades fluctuaban entre 17 y 29 años con una media de 28.8, moda de 21 y una desviación estándar de 2. Los cuestionarios fueron administrados por los profesores responsables de cada uno de los grupos durante sus horas de clase a los cuales se les dieron instrucciones precisas de tomar nota de todas las dudas que surgieran durante la administración tanto de la forma como del contenido del cuestionario.

\section{Procedimiento}

Finalmente, el CMEA fue administrado a los 1140 estudiantes a los que se les explicó el propósito del estudio y se les solicitó su participación voluntaria. Las instrucciones dadas a los estudiantes fueron leídas por la responsable de la investigación, directamente del formato del cuestionario. El tiempo medio de aplicación fue de 20 minutos. Asimismo, en el cuestio- 
nario se incluyó una pregunta para identificar a los estudiantes que deseaban conocer sus resultados para posteriormente enviarles, a través de su profesor, un informe con los mismos.

La administración del instrumento se realizó en las instalaciones de las facultades, concretamente en las aulas de clase de los estudiantes y por los propios profesores de grupo, durante el horario habitual de sus clases, ya que, como mencionan García y Pintrich (1991), se espera que la presencia de los compañeros, el profesor, los libros del curso y los materiales, estimulen a los sujetos a pensar acera de sus creencias actuales y su conducta para ese curso.

\section{Análisis Estadístico}

Una vez recopilada la información del CMEA, primero se realizó un análisis descriptivo de las variables de género, edad, titulación y curso, y se procedió a realizar los análisis respectivos. A continuación se realizó un análisis factorial de la estructura de cada una de las subescalas, una estimación de los errores asociados a las cargas factoriales, un cálculo de la correlación de cada ítem con el total de la subescala a la que pertenece el ítem, sin incluirlo. Así mismo se estimó el índice de fiabilidad Alpha de Cronbach para cada subescala y para las Escalas de Motivación y de Estrategias del Aprendizaje totales. Todos los análisis estadísticos fueron realizados mediante el programa SPSS (Statistical Package for Social Sciences) para Windows, versión 15.0.

\section{Resultados}

\section{Validez de contenido}

Dado que los 81 reactivos que conformaron el formato final del Cuestionario de Motivación y Estrategias de Aprendizaje (CMEA) fueron traducidos y adaptados al español a partir del MSLQ (Motivated Strategies for Learning Questionnaire) en su versión original en inglés y retraducidos nuevamente siguiendo las recomendaciones de la International Test Commission (ITC), se consideró que este procedimiento aseguraría la validez de contenido de los mismos con el fin de garantizar una adecuada inclusión en la forma definitiva del instrumento, sobre todo debido al análisis de contenido que se realizó de ellos para poder traducirlos y adaptarlos al contexto educativo de la muestra de estudiantes mexicanos. 
Sin embargo, se decidió explorar la validez de contenido de los ítems ya traducidos mediante el procedimiento de valoración de jueces expertos, para lo cual, se solicito a diez profesores y/o investigadores universitarios con experiencia y reconocimiento en el área de procesos y teorías de aprendizaje y/o psicometría su participación en esta fase del proyecto. Se explicó a cada uno de ellos por separado que como parte de un proyecto de investigación se estaba realizando una fase de validación de los ítems de un cuestionario de diagnóstico acerca de las estrategias de aprendizaje y la orientación motivacional de los estudiantes universitarios para lo cual se requería que valorara de forma cuidadosamente el contenido de cada enunciado en cuanto a su relevancia y pertinencia como ítem para medir el constructo original y por lo tanto su pertenencia a la subescala. Se le proporcionó a cada uno de los jueces expertos, un juego de tarjetas que contenían enunciados de los ítems traducidos pertenecientes a cada subescala; una muestra del tipo de reactivos que pertenecían a la subescala y la definición conceptual de cada una de las subescalas para guiar su decisión.

Los jueces expertos participaron en la valoración y clasificación de ítems dentro de las dimensiones teóricas, como una variante adaptada de la técnica Q-sorting. Se definió como criterio de aceptación un acuerdo interjueces mayor al $80 \%$ en la valoración y clasificación de ítems. Además del porcentaje de acuerdo interjueces, se estimó el índice de congruencia ítemdimensión de Hambleton y Rovinelly (1986). En términos generales, existió un acuerdo general de más del $90 \%$ de los jueces expertos en que los reactivos traducidos eran un indicador de medida del constructo de la subescala a la que pertenecían.

Como resultado del procedimiento anterior, y una vez que los ítems fueron traducidos, re-traducidos, adaptados y validados por los jueces expertos, se diseñó el cuestionario final en formato de autoinforme integrado por los 81 reactivos para ser probado en una fase piloto con una muestra de estudiantes universitarios. Se conservó la estructura de la versión original en inglés en relación con las quince subescalas y la ubicación de cada uno de los 81 ítems.

\section{Validez factorial}

Bajo el supuesto de que cada una de las 15 subescalas mide un constructo único y diferentes entre sí, se corrieron análisis factoriales por factorización de ejes principales, dada la falta de normalidad de las respuestas a los reactivos, a fin de determinar si este supuesto se cumplía y las cargas factoriales de los reactivos con este factor teórico único. En toda ocasión 
se estimó el índice de Kaiser-Meyer-Olkin (KMO) para comprobar la susceptibilidad de los datos a este tipo de análisis, así como una prueba de esfericidad de Bartlett a fin de evitar soluciones espurias. Todos las subescalas obtuvieron estadísticos adecuados en estas pruebas para poder confiar en sus análisis factoriales. A continuación se presentan los resultados de los análisis factoriales resumidos en la Tabla 3 y Tabla 4. En todos los casos los reactivos se asociaron a su factor principal con cargas factoriales mayores a 0.4 .

Tabla 3. Adecuación para el análisis factorial (KMO) y varianza explicada tras ajustar un modelo de análisis factorial con un factor único agrupando a los reactivos

\begin{tabular}{lccc}
\hline \multicolumn{1}{c}{ Escala de Motivación } & No. de reactivos & KMO & \% Varianza explicada \\
\hline Orientación a metas intrínsecas & 4 & 0.689 & 32.2 \\
Orientación a Metas extrínsecas & 4 & 0.719 & 36.6 \\
Valor de la tarea & 6 & 0.889 & 56.6 \\
Creencias de Control & 4 & 0.689 & 23.3 \\
Autoeficacia para el aprendizaje & 8 & 0.884 & 43.6 \\
Ansiedad ante los exámenes & 5 & 0.734 & 35.6 \\
Total & 31 & & \\
\hline
\end{tabular}

Tabla 4. Adecuación para el análisis factorial (KMO) y varianza explicada tras ajustar un modelo de análisis factorial con un factor único agrupando a los reactivos

\begin{tabular}{lccc}
\hline Escala de Estrategias de Aprendizaje & No. de reactivos & $K M O$ & \% Varianza explicada \\
\hline Repetición & 4 & 0.709 & 38.0 \\
Elaboración & 6 & 0.768 & 34.0 \\
Organización & 4 & 0.694 & 45.2 \\
Pensamiento crítico & 5 & 0.768 & 39.7 \\
Autorregulación metacognitiva & $9 *$ & 0.828 & 28.7 \\
Admón. tiempo y ambiente & $6^{*}$ & 0.730 & 21.3 \\
Regulación del esfuerzo & 4 & 0.682 & 22.3 \\
Aprendizaje con compañeros & 3 & 0.610 & 32.3 \\
Búsqueda de ayuda & $3 *$ & 0.637 & 30.2 \\
Total & 44 & & \\
\hline
\end{tabular}

* Nota: Se eliminaron algunos reactivos con poca asociación con el resto de la subescala a fin de elevar sus índices psicométricos 


\section{Fiabilidad}

La consistencia interna del cuestionario fue obtenida mediante el índice de fiabilidad Alpha de Cronbach para cada una de las 15 subescalas y para la Escala de Motivación y de Estrategias por separado. Las Tablas 5 y 6 muestran el número de reactivos y los índices de confiabilidad Alpha de Cronbach de cada una de las subescalas de la sección de Motivación y de Estrategias de Aprendizaje respectivamente y de la escala total.

Tabla 5. Índices de confiabilidad Alpha de Cronbach y número de reactivo de la Escala de Motivación por subescala

\begin{tabular}{lcc}
\hline \multicolumn{1}{c}{ Escala de Motivación } & No. De reactivos & $\begin{array}{c}\text { Índice Alpha de } \\
\text { Cronbach }\end{array}$ \\
\hline Orientación a Metas Intrínsecas (OMI) & 4 & .65 \\
Orientación a Metas Extrínsecas (OME) & 4 & .65 \\
Valor de la Tarea (VT) & 6 & .87 \\
Creencias de Control (CC) & 4 & .52 \\
Autoeficacia para el aprendizaje (AEPA) & 8 & .85 \\
Ansiedad ante los exámenes (AE) & 5 & .72 \\
Total & 31 & .88 \\
\hline
\end{tabular}

Tabla 6. Índices de confiabilidad Alpha de Cronbach y número de reactivos de la Escala de Estrategias de Aprendizaje por subescala

\begin{tabular}{lcc}
\hline \multicolumn{1}{c}{ Escala de Estrategias de Aprendizaje } & No. de reactivos & $\begin{array}{c}\text { Índice Alpha de } \\
\text { Cronbach }\end{array}$ \\
\hline Repetición (REP) & 4 & .71 \\
Elaboración (ELA) & 6 & .72 \\
Organización (ORG) & 4 & .72 \\
Pensamiento crítico (PC) & 5 & .76 \\
Autorregulación metacognitiva (ARM) & $9^{*}$ & .77 \\
Admón. del tiempo y del ambiente (ATA) & $6^{*}$ & .65 \\
Regulación del esfuerzo (RE) & 4 & .48 \\
Aprendizaje con compañeros (AC) & 3 & .56 \\
Búsqueda de ayuda (BA) & $3^{*}$ & .43 \\
Total & 44 & .90
\end{tabular}

* Nota: Se eliminaron algunos reactivos con poca asociación con el resto de la subescala a fin de elevar sus índices psicométricos 
Los índices de consistencia interna (Alfa de Cronbach) mostrada en la Tabla 5 correspondientes a la Escala de Motivación, fluctuaron entre .87 para la subescala de VT y .52 para la subescala de CC. El índice Alfa de Cronbach de la escala total fue de .88. El resto de las subescalas obtuvieron índices mayores de .65 bastante bueno para este tipo de cuestionarios. En el caso de la Escala de Estrategias de Aprendizaje que se muestra en la Tabla 6 los índices de consistencia variaron entre .77 para la escala de ARM hasta .43 en el caso de la subescala de BA. El índice de consistencia interna de la Escala de Estrategias fue de .90. En este caso se destaca el resultado de las subescalas de estrategias de aprendizaje (REP, ELA, ORG Y PC) y la subescala de ARM que resultaron con índices mayores de .70.

\section{Discusión y conclusiones}

En este estudio, se reporta el procedimiento de traducción y adaptación al contexto educativo mexicano, de uno de los cuestionarios de autorreporte más utilizados en los ambientes educativos para evaluar la motivación y el uso de estrategias cognitivas, metacognitivas y de contexto: el Motivated Strategies for Learning Questionnaire (MSLQ). La cuidadosa traducción de los ítems al español de México, su adaptación al contexto educativo de la muestra de estudiantes mexicanos, así como la rigurosa administración del instrumento, todo ello orientado a asegurar que dichas condiciones no constituyeran una fuente de error respecto del test original, redundó en la confirmación de la estructura factorial original del instrumento de 6 subescalas de motivación y 9 de estrategias de aprendizaje, así como en los índices de consistencia interna, que resultaron satisfactorios.

Los resultados obtenidos en relación a la validez factorial, reprodujeron la estructura factorial bajo la cual se asienta el MSLQ (Pintrich, Smith, García y McKeachie, 1991,1993) y ahora el CMEA. Además, muestran que los ítems fueron agrupados en cada uno de los factores mediante la factorización de ejes principales, lo cual demuestra la unidimensionalidad de las subescalas asociándose los reactivos de manera adecuada. Los resultados anteriores muestran discrepancia con los reportados por otros autores en contextos educativos diferentes (Cardoso, 2008; Martínez y Galán, 2000; Roces, Tourón y González, 1995). El resultado anterior debe ser tomado con cautela ya que incluso García y McKeachie (2005) reportan que con el MSLQ, administrado a diferentes poblaciones como estudiantes de bachillerato o de universidad, emergen estructuras factoriales diferentes (Pintrich y De Groot, 1990a ; Pintrich et. al 1991) pero los resultados encajan dentro del modelo conceptual general y recomiendan que 
para futuras investigaciones, es necesario abordar estas diferencias para determinar, si son el resultado de la variación del método o realmente reflejan diferencias en el desarrollo de la motivación y la cognición (García y McKeachie, 2005).

De igual manera, todo el proceso de construcción y adaptación de los ítems al contexto educativo mexicano, influyó positivamente la fiabilidad de la información, ya que al comparar la consistencia interna de algunas de las subescalas, resultó mejor que algunas reportadas en contextos anglosajones (Pintrich, Smith, García y McKeachie, 1991,1993), hispanos (Roces, Tourón, y González, 1995; Martínez y Galán, 2000), latinos (Cardozo, 2008) y chinos (Sachs, Law, \& Chan, 2001). Por otra parte, y en términos generales, los resultados de los índices de consistencia interna obtenidos con el CMEA fueron aceptables y bastante semejantes a los reportados para el MSLQ e incluso en algunas subescalas fueron superiores. Ejemplo de lo anterior es el caso de la subescala OME, donde el índice del CMEA fue $r=.65$ y el del MSLQ $r=.62$. Para la subescala de REP, $r=.71$ y MSLQ $r=.69$ y ORG, $r=.72$ y MSLQ $r=.64$ respectivamente (García y McKeachie, 2005). Reconociendo las diferencias socio-económicas y culturales entre las poblaciones objetivo de ambos estudios y sin caer en la tentación de comparar los resultados al momento de su interpretación, la comparación anterior se justifica dado que las características psicométricas de los instrumentos son similares en cuanto al constructo que se pretende medir y la estructura interna de la escala que sirvió de base para el desarrollo del instrumento.

En el momento presente, en el Nivel Medio Superior y Superior de educación de México, se ha iniciado un transformación orientada al logro de perfiles de egreso en términos de competencias para el aprendizaje autónomo por lo cual, el CMEA representa una alternativa valiosa para su medición, evaluación y posterior intervención en el desarrollo de las competencias para el aprendizaje autónomo y autorregulado de los estudiantes, (Martín, Bueno y Ramírez, 2010)

Además, un instrumento que evalúe la motivación, las estrategias autorregulatorias y de aprendizaje, así como la gestión del contexto de aprendizaje por parte de los estudiantes universitarios, permitirá proporcionar información valiosa a las áreas de atención a estudiantes para apoyarlos en su proceso de aprender a aprender que se ha establecido como uno de los objetivos de la educación universitaria contemporánea, para que cada día en las aulas de 
clase sea una nueva experiencia que promueva en el estudiante una conducta académica autónoma y autorregulada.

Los resultados obtenidos en el presente estudio, apoyan el uso del CMEA como una medida valida y confiable de la motivación y el uso de estrategias de aprendizaje de los estudiantes en el contexto educativo mexicano. Sin embargo, es necesario comentar que como todos los cuestionarios de autorreporte el CMEA posee limitaciones y requiere de nuevos estudios mediante análisis factorial confirmatorio y con muestras de estudiantes aún mayores y de diferentes niveles educativos con el objetivo de obtener información psicométrica más solida. En cuanto a los ítems eliminados que no cargaron en ninguno de los factores propuestos, es necesario revisar su redacción y volver a probarlos en futuras investigaciones a fin de probar su consistencia interna con el resto de ítems de la subescala a la que pertenecen, para poder utilizarlos en posteriores aplicaciones el cuestionario.

Se sugiere continuar con estudios que exploren de forma más minuciosa, las propiedades psicométricas del instrumento mediante métodos estadísticos actuales, hasta conseguir establecer de manera fiable y válida la estructura factorial y la consistencia interna del CMEA para utilizarlo en estudios más amplios y con distintas poblaciones e incluso con distintas modalidades de enseñanza como la educación a distancia. Los resultados reportados en esta investigación, muestran una gran potencia del instrumento de proporcionarnos información útil para contar con un instrumento de medida que nos permita encontrar respuesta a la pregunta inicial de los trabajos de autorregulación del aprendizaje: ¿Cómo los estudiantes llegan a ser expertos en sus propios procesos de aprendizaje?

\section{Referencias}

Boekaerts, M., Pintrich, P.R. y Zeidner, M. (2000). Handbook of Self-Regulation. San Diego: Academic Press.

Brown, W. y Holtzman, W. (1967). Survey of study habits and attitudes. NY: Psychological Corporation.

Cardozo, A. (2008). Motivación, aprendizaje y rendimiento académico en estudiantes del primer año universitario. Laurus, 14, 209-237.

Christensen, F. A. (1968). College adjustment and study skills inventory. Berea, OH: Personal Growth Press. 
Cleary, T., Zimmerman, B. J. y Keating, T. (2006). Training physical education students to self-regulate during basketball free-throw practice. Research Quarterly for exercise and sport, 77, 251-262.

Dunn, K. E., Lo, W. J., Mulvenon, S. W. y Sutcliffe, R. (2012). Revisiting the Motivated Strategies for Learning Questionnaire. A Theoretical and Statistical Reevaluation of the Metacognitive Self-Regulation and Effort Regulation Subscales. Educational and Psychological Measurement, 72(2), 312-331.

Goldman, R. y Warren, R. (1973). Discriminant analysis of study strategies connected with college grade success in different major fields. Journal of Educational measurement, $10,39-47$.

Greene, J. A. y Azevedo, R. (2007). Adolescents' use of self-regulatory processes and their relation to qualitative mental model shifts while using hypermedia. Journal of Educational Computing Research, 36, 125-148.

García, T. y McKeachie, W. (2005). The making of the Motivated Strategies for Learning Questionnaire. Educational Psychologist. 40 (2), 117-128.

García, T. y Pintrich P. R. (1991). Student motivation and self regulated learning: A LISREL model. Paper presented at the Annual meeting of the American Research Association, Chicago, IL.

Limón, M. (2004). (Coor.). En homenaje a las contribuciones de Paul R. Pintrich a la investigación sobre Psicología y Educación. Electronic Journal of Research in Educational Psychology, 2 (1), 157-210.

Hambleton, R. y Rovinelli, R. (1986). Assessing the dimensionality of a set of test items. Applied Psychological Measurement. 10 (3), 287-302.

Lockhart, D. y Schmeck, R. (1984). Learning styles and classroom evaluation methods: Different strokes for different folks. College Student Journal, 17, 94-100.

Martín, M.E., Bueno, J.A. y Ramírez, M.C. (2010). Evaluación del aprendizaje autorregulado en estudiantes de bachillerato mexicanos. Aula abierta, 38, (1), 59,70.

Martínez, J. R., y Galán, F. (2000). Estrategias de aprendizaje, motivación y rendimiento académico en alumnos universitarios. Revista Española Orientación y Psicopedagogía, 11(19), 35-50.

Muñiz, J. y Hambleton, R. (1996). Directrices para la traducción y adaptación de los test. Papeles del Psicólogo, 66, 1-5. 
Núñez, J.C., Solano, P., González-Pienda, J.A. y Rosario, P. (2006). Evaluación de los procesos de autorregulación mediante autoinforme. Psicothema, 18 (3), 353-358.

Ommundsen, Y. (2006). Pupils' self-regulation in physical education: the role of motivational climates and differential achievement goals. European Physical Education Review. $12(3), 289-315$.

Paris, S. G.; Lipson M. Y. y Wixson, K. K. (1983). Becoming a strategic reader. Contemporary Educational psychology, 8, 293-316.

Pintrich, P. R. (1989). The dynamic interplay of student motivation and cognition in the college classroom. En: C. Ames y M. Maehr (Eds.) Advances in motivation and achievement (Vol. 6). Motivation enhancing environment (pp. 117-160). Greenwich, CT: JAI Press.

Pintrich, P. R. y De Groot, E. V. (1990a). Motivational and self regulated learning components of classroom academic performance. Journal of Educational Psychology, 82 (1), 33-40.

Pintrich, P. R. y De Groot, E. V. (1990b). Individual differences in student motivational orientation, self regulated learning and academic achievement. Paper presented at the International Congress of Applied Psychology.

Pintrich, P. R., Smith, D. A., García, T. y McKeachie, W. J. (1991). A manual for the use of the Motivated Strategies for Learning Questionnaire (MSLQ). Ann Arbor, MI: NCRIPTAL: The University of Michigan.

Pintrich, P. R., Smith, D. A., García, T. y McKeachie, W. J. (1993). Reliability and predictive validity of the motivated strategies for learning questionnaire (MSLQ). Educational and Psychological Measurement, 53, 801-803.

Roces, C., Tourón, J., y González, M.C. (1995). Validación preliminar del CEAM II (Cuestionario de Estrategias de Aprendizaje y Motivación II). Psicológia, 16(3), $347-366$.

Sachs, J., Law, Y. K., Chan, C. K. K., y Rao, N. (2001). A nonparametric item analysis of the Motivated Strategies for Learning Questionnaire-Chinese version. Psychologia, 44, 197-208.

Schunk, D. H., y Zimmerman, B. J. (1994).Self-regulation in education: retrospect and prospect. En D. H. Schunk y B. J. Zimmerman (Eds.), Self-regulation of learning and performance. Issues and educational applications. Hillsdale, NJ: Erlbaum. 
Schmitz, B. y Wiese, B. S. (2006). New perspectives for the evaluation of training sessions in self-regulated learning: time-series analyses of diary data. Contemporary Educational Psychology, 31, 64-96.

Schunk, D. H. (2005). Self-regulated learning: the educational legacy of Paul R. Pintrich. Educational Psychologist, 40 (2), 85-94.

Schunk, D. H. y Zimmerman, B. J. (Eds.). (2007). Motivation and self-regulated learning: Theory, research, and application. Mahwah, NJ: Lawrence Erlbaum.

Stoeger, H. y Ziegler, A. (2007). Evaluation of a classroom-based training to improve selfregulated learning: Which pupils profit the most? Manuscript submitted for publication.

Taylor, R. T. (2012). Review of the Motivated Strategies for Learning Questionnaire (MSLQ) Using Reliability Generalization Techniques to Assess Scale Reliability. Auburn University.

Torrance, E. P., Reynolds, C. R., Riegel, T. y Ball, O. (1977). Your style of learning and thinking: Forms A and B. The Gifted Child Quarterly, 21, 563-573.

Van Den Hurk, M. (2006). The relation between self-regulated strategies and individual study time, prepared participation and achievement in a problem-based curriculum. Active Learning in Higher Education. 7 (2), 155 - 169.

Weinstein, C. E., Schulte, A. C. y Palmer, D. R (1987). Learning and study strategies inventory. Clearwater, FL: $\mathrm{H}$ y $\mathrm{H}$ Publishing.

Weinstein, C. E. y Underwood, V. L. (1985). Learning strategies: The how of learning. En: J. W. Segal, S. F. Chipman y R. Glaser (Eds.), Thinking and learning skills: Relating instructions to research (Vol. 1, pp. 241-258). Hilldale, NJ: Erlbaum.

Winne, P. H., Nesbit, J. C., Kumar, V., Hadwin, A. F., Lajoie, S. P., Azevedo, R., et al. (2006). Supporting self-regulated learning with study software: The learning kit project. Technology, Instruction, Cognition and learning. 3, 105-113.

Zimmerman, B. J. (1986). Becoming a self regulated learner: Which are the key subprocesses? Contemporary Educational Psychology, 11, 307-313.

Zimmerman, B. J. (1989). A social cognitive view of self-regulated academic learning. Journal of Educational Psychology, 81 (3), 329-339.

Zimmerman, B. J. (1994) Dimensions of academic self-regulation: a conceptual framework for education. En D. H. Schunk y B. J. Zimmerman (Eds.), Self-regulation of 
learning and performance. Issues and educational applications. Hillsdale, NJ: Erlbaum.

Zimmerman, B. J. (2008). Investigating Self-Regulation and Motivation: Historical Background, Methodological Developments, and Future Prospects. American Educational Research Journal. 45(1), 166 - 183.

Zimmerman, B. J. y Schunk, D. H. (1989). Self-regulated learning and academic achievement. Theory, research and practice. NY: Springer-Verlag.

Zimmerman, B. J. y Schunk, D. H. (2001). Reflections on theories of self-regulated learning and academic achievement: Theoretical Perspectives. Mahwah, NJ: Lawrence Erlbaum.Gadner, H. (1993). Multiple intelligences. The theory in practice. New York: Basic Books. 